\title{
Modernización, dependencia y marginalidad: itinerario conceptual de la sociología latino- americana'
}

ALEXIS GORTÉS M*

\section{Resumen}

El objeto de este artículo es analizar y discutir las continuidades y superaciones de tres teorías fundamentales del pensamiento sociológico latinoamericano: la Teoría de la Transición a la Modernidad de Gino Germani, la Teoría de la Dependencia de Fernando Henrique Cardoso e Enzo Faletto y la Teoría de la Marginalidad de Roger Vekemans. Pese a las críticas que diagnostican su superación, se mostrará cómo nuevas tendencias teóricas están dando continuidad al debate sociológico latinoamericano iniciado en el siglo pasado. Así, por ejemplo, se afirma que la Teoría de Sistemas podría revivir tópicos de la contribución germaniana por la noción de transición permanente implícita en el proceso de especialización funcional de ambas. Por otro lado, el proceso de globalización en parte actualizaría la discusión propia de la Dependencia sobre soberanías nacionales y nuevas formas de subordinación internacional. Y, finalmente, las Teorías sobre la Exclusión Social nacerían debatiendo en continuidad y ruptura con las Teorías de la Marginalidad.

Palabras clave: Modernidad. Desarrollo/sub-desarrollo. Dependencia. Marginalidad. Sociología latinoamericana.

\footnotetext{
1 Una versión preliminar de este trabajo fue presentado en el Grupo de Trabajo $n^{\circ} 17$ de Pensamiento Latinoamericano y Teoría Social en el XXVII Congreso de la Asociación Latinoamericana de Sociología (ALAS). Buenos Aires, agosto de 2009.

* El autor es doctorando en Sociología por el Instituto de Estudos Sociais e Políticos da Universidade Estadual do Rio de Janeiro (Brasil) y becario do Conselho Nacional de Desenvolvimento Científico e Tecnológico (CNPq) - Brasil. E-mail: cortes.alexis@gmail.com
} 


\section{Modernization, Dependency and Marginality: conceptual itinerary of Latin American sociology}

\section{Abstract}

This paper aims to analyze and discuss the continuities and improvements of three Latin American fundamental social theories: the theory of transition to modernity as formulated by Gino Germani, the dependency theory developed in the works of Cardoso and Faletto, and the Marginality Theory by Roger Vekeman. In spite of the critiques that point the superseding of these theories, this article will expose new theoretical trends towards the endurance of the Latin American sociological debate initiated in the 20th century. Thus, it is stated that the Systems Theory could reintroduce topics of Germani's contribution through the idea of permanent transition implied in the processes of functional specialization present in both theories. The globalization process, in turn, would update the debate, intrinsic to dependency theory, on national sovereignties and new forms of international subordination. Finally, the theories of social exclusion would arise both continuing and breaking with marginality theories.

Keywords: Modernity. Development/underdevelopment. Dependency. Marginality. Latin American sociology.

\section{Introducción}

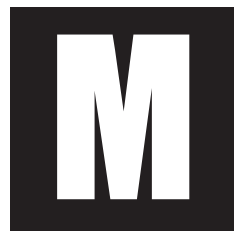

odernización, subdesarrollo/desarrollo, transición, dependencia, centro/periferia, marginalidad. Sin duda, estas categorías podrían sintetizar buena parte de los debates que marcaron la trayectoria de consolidación de la sociología latinoamericana durante el siglo XX. Precisamente el objeto de este artículo es discutir las continuidades y superaciones de tres teorías que construyeron sus andamiajes analíticos mediante estos conceptos y que, por lo demás, fueron indispensables para el desarrollo de la reflexión sociológica en nuestro continente: la Teoría de la Transición a la Modernidad de Gino Germani, La Teoría de la De- 
pendencia de Fernando Henrique Cardoso y Enzo Faletto y la Teoría de la Marginalidad de Roger Vekemans.

No obstante el papel central que estas teorías cumplieron en la evolución de la sociología latinoamericana, por estos días se puede percibir una tendencia, por parte de algunos de los nuevos esfuerzos de reflexión teórica, que marca la ruptura y superación de las nuevas tendencias frente a esta "vieja sociología" continental. En contraste, este artículo se posicionará enfatizando más bien la continuidad entre la tradición teórica de los autores mencionados y algunas de las nuevas perspectivas que marcan los debates teóricos en este lado del globo.

Para ello, sin embargo, es necesario dejar en claro qué se entenderá por ruptura entre la "vieja" y la "nueva" teoría sociológica. Es evidente que todas las perspectivas teóricas actuales inician su reflexión a partir de un contexto completamente diferente al de la "vieja sociología" latinoamericana, pues han operado una serie de transformaciones en el escenario continental y mundial. Entre otros, ha aumentado la complejidad social y han emergido nuevos fenómenos aparentemente ajenos a las teorías que ocuparon un papel protagónico durante el siglo pasado. En otras palabras, las tres teorías que revisaremos son construcciones que enfrentan las limitaciones de su propio tiempo. No obstante, no es del interés de este texto enumerar más o menos detalladamente las superaciones de las mismas por las restricciones impuestas por su propia temporalidad. O sea, si existe un quiebre entre "la vieja" y "la nueva" teoría, éste no está dado apenas por el carácter datado de la primera, sino por diferencias sustantivas entre una y otra.

Es por esto que tomaremos como marco de ruptura la perspectiva elaborada por Chernilo y Mascareño (2005), quienes afirman que este quiebre es de carácter programático. Para estos autores, la debilidad del pensamiento sociológico sub-continental frente a los desafíos propuestos 
por la globalización y el cosmopolitismo estaría dada por tres obstáculos epistemológicos: uno de carácter estructural, que llevó a la comprensión de América Latina como una versión limitada e incompleta de la modernidad europea; uno normativo, que lo llevó a caer en el nacionalismo metodológico o a la búsqueda de un ethos particularista; y finalmente uno identificado con la falta de autonomía del quehacer sociológico, el que se vuelve una mera tecnología de transformación social al servicio de particularismo políticos y de actores sociales determinados.

Será considerando y tematizando estas proposiciones que se afirmará la continuidad del pensamiento sociológico latinoamericano. Por ello, el artículo será dividido en tres partes principales, en cada una de ellas se expondrá sintéticamente los principales conceptos y cuestionamientos de cada teoría. Finalmente el texto será cerrado con una reflexión en la que se enfatizarán aquellos elementos de ruptura y continuidad que indiquen vigencia o superación de las diferentes teorías, mostrando posibles formas en las que se expresaría la actualidad de las mismas.

\section{Gino Germani y la transición a la modernidad}

Conocido como el fundador de la sociología argentina (Izaguirre, 2005), Gino Germani fue también el autor de unas de las teorías de la modernización más originales del siglo pasado. Con ella, este autor ítalo-argentino, intentó explicar el paso de una sociedad tradicional a una sociedad industrial en América Latina. Bajo la lógica de esta teoría, nuestro continente era caracterizado como una sociedad que experimentaba fuertes y aceleradas transformaciones, donde el trazo fundamental era el paso de una sociedad tradicional a una desarrollada. En esta transición sería posible observar la coexistencia de formas sociales pertenecientes a diferentes épocas. Lo que implicaría un alto grado de conflictividad por la 
continua ruptura con el pasado, generando consecuencias en las instituciones, en los grupos sociales y en la conciencia de las personas (Germani, 1973b). Este fenómeno era denominado por el autor como la teoría del "dualismo estructural", el que se entendía como la coexistencia en una misma sociedad de dos o más formas estructurales dadas, en tanto "tipos ideales". Esto se debería a la existencia de "asincronías" en los procesos de cambio social, es decir, descompases, ajustes y atrasos (en relación a cambios esperados para una determinada estructura) en diferentes dimensiones de la vida social (culturales, motivacionales, políticas, etc.). La asincronía implicará la convivencia de lo que Germani entendía como formas declinantes (sobrevivencias tradicionales), básicas (configuraciones modernas en el presente) y formas emergentes (orientaciones que anticipan un estado futuro de la sociedad moderna).

Para este autor (Germani, 1971), el requisito universal mínimo para la existencia de cualquier sociedad industrial-moderna era la secularización de todas las dimensiones de la vida social. Específicamente: I) el predominio de la "acción electiva" (las acciones humanas se sustentan en la realización de elecciones individuales), que substituye un marco normativo determinado por una "acción prescriptiva"; II) la especialización creciente de las instituciones y la emergencia de sistemas de valores específicos y relativamente autónomos para cada esfera institucional; III) así como la institucionalización creciente del "cambio", el que pasa a ser previsto por las propias normas que la sociedad se da para existir.

Un recurso analítico empleado por el autor para comprender este proceso de secularización fue la aplicación de las "variables-pauta" de Parsons, probablemente uno de los elementos por el que es más conocido y a la vez simplificado. Así, en las sociedades tradicionales predominarían los papeles adscritos, difusos, particularistas y afectivos; mientras que en 
la sociedad industrial se vuelven más relevantes los de tipo universalista, de desempeño, específicos y afectivamente neutros ${ }^{2}$.

La transición a la modernidad no se produciría linealmente ni sin conflicto, al contrario, las asincronías serían un rasgo universal de la transformación social, sobre todo cuando es un cambio que no es ni dirigido ni planeado. Las transiciones de los diferentes países variarían en velocidades y secuencias por las diferentes circunstancias históricas que cada sociedad debe enfrentar.

Es interesante destacar que la explicación causal de los cambios sociales no es interpretada por este autor epifenoménicamente, o sea, a partir de un único factor, por el contrario, asume una causalidad recíproca. Los procesos de desarrollo económico - el que es entendido como crecimiento autosustentable - condicionan los procesos de modernización social y política, tanto como estos últimos se condicionan entre sí o condicionan el propio desarrollo económico. De ahí que no resulte extraño encontrar en sus textos explicaciones que conjugan diferentes variables simultáneamente, a contracorriente de las tendencias deterministas reinantes en la época.

A pesar de la enorme influencia que Germani ejerció en su generación, fuertes críticas se ciñeron sobre sus principales postulados. Principalmente lo que se cuestionaba era el hecho de que su propuesta de transición de una sociedad tradicional para una moderna suponía situar a las sociedades industriales avanzadas como modelos o metas que inevitablemente serían alcanzados por los países atrasados, lo que implicaba desconsiderar los contextos históricos estructurales específicos de cada país. Asimismo, la dicotomía tradicional/moderno presumía necesariamente un origen y un destino dado, un "deber ser" del desarrollo con

2 Parsons también planteaba la existencia de una quinta variable: "Orientación para intereses privados versus orientación para intereses colectivos", sin embargo, Germani la descartó por no considerarla relevante para el análisis de la transición latinoamericana. 
fuertes ribetes normativos, cuya simplificación dicotómica envuelve un grado de deshistorización analítica que no considera la particularidad de cada país, pues la preocupación es la búsqueda de "completud" del modelo de modernización europeo (Larraín, 1989). Desde otra perspectiva, específicamente desde la trinchera del particularismo latinoamericano, se cuestionó el paradigma desarrollista - y a Germani con él - por no dar suficiente espacio a la cultura en su reflexión, considerando la modernización como una mera opción tecnológica, como una tecnología de la transformación social programada (Morandé, 1987).

En gran medida estas críticas, en mayor o menor grado, han hecho eco o han ayudado a reproducir la idea de que la propuesta de Germani es una mera aplicación de la teoría de la modernización parsoniana en versión latinoamericana. Este reduccionismo ha contribuido a obstaculizar la difusión y actualización del pensamiento germaniano ${ }^{3}$. Enhorabuena el trabajo de Alejandro Blanco (2003) ha hecho cada vez más difícil seguir sosteniendo esta visión simplificada, pues ha proporcionado bastante evidencia para mostrar que, no obstante la obra de Parsons resulta convergente con parte de la Teoría del Desarrollo germaniana, el uso efectivo de aquélla por Germani sería en un tono sumamente heterodoxo, siendo Parsons, por lo demás, una de las tantas fuente teóricas empleadas por el autor de Política y Sociedad en una época de transición, entre las que es posible encontrar influencias a todas luces contrarias al estructural-funcionalismo como Mead y otros críticos a esa escuela.

En esa misma línea, otros autores (Domingues e Maneiro, 2004) también han remarcado que la contribución de Germani no se reduce a una buena aplicación de la teoría parsoniana al continente, pues la arquitectura teórica de Germani estaba bien consolidada antes de la apli-

3 Por ejemplo (Acevedo Rodríguez, 2009) 
cación de las "variables-pauta". Es más, para estos autores, la novedad en la interpretación de la modernidad estaría dada por la aplicación del concepto de "acción electiva", pues implicará otorgar al concepto de libertad un lugar analítico central mucho antes de que otras teorías sociales la incorporasen a su acervo conceptual.

No está demás situar la contribución de Germani en el contexto de época en el que estaba inmerso. De hecho, la discusión sobre subdesarrollo/desarrollo y sus posibles trayectorias fue determinada fuertemente por la disputa de diferentes modelos de desarrollo que intentaban mostrar su superioridad como alternativas. La idea de "ventajas del atraso" es un buen ejemplo del espíritu y de la confianza en la posibilidad de modelar y dirigir la sociedad hacia un norte determinado que permitiese la superación de los obstáculos que truncaban el desarrollo del continente (Moore, 1975). La creencia era que los países en proceso de transición podían sacar lecciones de las experiencias de otros que ya hicieron el pasaje a la modernidad, dando origen a otras vías de desarrollo.

Por lo anterior, es justo recalcar que, para Germani, la transición a la modernidad no era un camino lineal hacia una industrialización que replicara la modernización europea. Para este autor no existiría un tipo único de sociedad industrial, por lo que consideraba necesario dejar en el análisis un amplio margen para variaciones estructurales y culturales, en coherencia con la noción de que los diversos subprocesos de las transformaciones tienen ritmos diferentes y con variadas secuencias (he aquí otra dimensión del concepto de asincronía). Los tipos opuestos de sociedad serían extremos de un continuum pluridimensional cuyas diferentes formas de transición pueden derivar en múltiples experiencias de sociedad. Así la dicotomía tradicional/moderna debe ser comprendida como tipos ideales analíticos y no históricos. Por ello, no obstante moderno parecía ser sinónimo de europeo u occidental, el desafío de nuestros países era, 
según sus mismas palabras, construir su propio modelo de "modernidad", de manera que no traicione su herencia cultural (Germani, 1973a, 81).

Un aspecto que puede ser explorado como un desafío de actualización del pensamiento de Germani es su posible rehabilitación - con las salvedades pertinentes - mediante el empleo de la Teoría de Sistemas de Luhmann. Efectivamente, la Teoría de Sistemas podría revivir tópicos de la contribución germaniana tanto por el trazo funcionalista en común, como por el hecho de que en la visión sistémica está contenida la idea de transitoriedad, por la descripción de la modernidad como un permanente proceso de diferenciación funcional de sistemas. Tal como lo expresa uno de los más importantes expositores latinoamericanos de la teoría de sistemas:

Desde Germani en adelante, es imposible no pensar en los dilemas de la diferenciación funcional latinoamericana. Su teoría de la asincronía - en que en cuentas resumidas describe niveles de desarrollo diferenciados de las instituciones de la modernidad clásica latinoamericana- es una de las fórmulas que mejor ilustran la plasticidad del proceso en el marco de la transición entre tradición y modernidad. (Mascareño, 2004, p. 68)

Un elemento negligenciado en este texto acerca de la obra de Germani es su análisis del populismo (argentino principalmente) y sus reflexiones sobre la conformación de regímenes autoritarios en nuestro continente. Aunque esta ausencia en parte se explica por la prioridad dada al flanco más criticado de la producción teórica germaniana - y que, por lo demás, es su núcleo central - bien vale la pena hacer eco de las palabras de Gonzalo Varela, en relación a la originalidad y centralidad de las contribuciones de Germani en este ámbito.

Independientemente de la aridez y el abstraccionismo que se han reprochado al funcionalismo - y que aparecen en la obra de Germani - y más allá de aspectos discutibles o anacrónicos, su lectura sigue siendo provechosa como 
ejemplo metodológico de construcción de una teoría y, en particular, por sus análisis del populismo, que en buena parte se mantienen en pie de igualdad si no de superioridad (tal vez de paternidad) con los de otros autores. (Várela Petito, 2008, p. 243)

\section{Cardoso y Faletto y la Dependencia Latinoamericana}

Para Domingues (2007), la trayectoria de la sociología de la modernización de América Latina se caracteriza por el paso de explicaciones endógenas del subdesarrollo para la relación con planos externos y con factores exógenos como marco explicativo de sus interpretaciones. Este paso sería uno de los aportes de las teorías de la CEPAL y de las teorías de la dependencia.

La teoría cepaliana del desarrollo económico estaba inspirada en el trabajo del economista argentino Raúl Prebisch y apuntaba a la necesidad de un "crecimiento hacia dentro", mediante un proceso de sustitución de importaciones, en contraposición al "crecimiento hacia fuera" basado en la exportación de materias primas. Un elemento central en esta teoría era la existencia de un ordenamiento mundial en el que las naciones del "centro" desarrollado mantienen a las naciones de la "periferia" en una condición de dependencia (Prebisch, 1947).

No obstante el clima intelectual y político favorable a las ideas anteriores, el deterioro de las exportaciones de materias primas, así como del proceso de industrialización mediante substitución de importaciones, que resultaron en una pérdida de dinamismo de las economías latinoamericanas, inauguraron un periodo de pesimismo y crítica a las teorías de la modernización y, en particular, a la efectividad de las políticas cepalianas. Desde el centro de estas críticas emergieron las nuevas teorías de la dependencia a mediados de los años 1960. 
Si bien no es posible hablar de una teoría de la dependencia, la elaborada por Cardoso y Faletto (2003) es considerada como la más sólida, balanceada y completa de todas ellas. Para Larraín (1989) tendría dos características fundamentales: primero su carácter crítico y provisorio, pues, no obstante su cuestionamiento al desarrollismo por no haberse dado el "salto adelante" que se esperaba después de la II dependencia tendrá una cierta continuidad con el desarrollismo - por la centralidad de categorías como centro/periferia o de relaciones asimétricas. El segundo rasgo será la integración dialéctica de aspectos sociológicos y políticos para pensar los procesos de desarrollo económico, lo que representará un quiebre con los análisis unilaterales que simplemente yuxtaponían variables para comprender la modernización.

Estos dos autores van a criticar la idea de "subdesarrollo", proponiendo el concepto de "dependencia", la que sería un trazo particular de la instauración del modo capitalista en las economías periféricas, en donde, en último término, las decisiones que afectan la economía son tomadas en función de la dinámica y de los intereses de las economías centrales. Así, lo específico de la situación de "subdesarrollo" nace de la relación entre sociedades periféricas y centrales, en donde se vincularán en un mismo mercado economías con distintos niveles de diferenciación de sus sistemas productivos. La dependencia no es una cuestión temporal o de etapas a ser superadas, sino una cuestión posicional o funcional a la estructura de la división internacional del trabajo.

La dependencia no es antagónica, bajo esta lógica, con la noción de desarrollo, pues sería posible incrementar los niveles de desarrollo de un país y mantener, a la vez, los lazos de dependencia que la subordinan internacionalmente. La dependencia no es tampoco sólo una causa externa, pues es una condición general que sólo se expresa a sí misma mediante los conflictos internos de cada país. Esto, porque la dependencia 
tendría un carácter asociado, no siendo funcional sólo a la hegemonía internacional, sino también a la específica forma en cómo la subordinación es recibida por las clases dominantes locales.

Así, cuando ocurren cambios en el mundo capitalista, no se producen transformaciones "replicadas" por igual en la periferia. Por eso es que los autores prefieren hablar de "situaciones de dependencia" más que de una teoría de la dependencia. La subordinación al centro no es una recepción pasiva, sino una negociación, aceptación, adaptación o rechazo por parte de la política interna y el sistema de clases de un país o región periférico. Lo que se enfatiza aquí es la naturaleza política de los procesos de transformación económica, siendo el problema teórico fundamental la determinación de los modos que adoptan las estructuras de dominación local. Por un lado, el interés está en las luchas políticas entre grupos y clases y, por otro, en la historia de las estructuras económico-políticas de dominación internas y externas.

De esta manera, la dependencia no puede ser considerada como una situación estable o permanente, pues puede cambiar y ser cambiada. Así como no puede ser vista como una condición homogénea, pues depende de la situación específica de cada país y de las alianzas de clases nacionales. Así la teoría es cuidadosa y no busca elaborar un concepto abstracto y totalizante que pueda substituir el análisis concreto y real.

Lo anterior podría llevar apresuradamente a una acusación de "nacionalismo metodológico", pues el diagnóstico de la dependencia es global, pero su explicación final es nacional (Chernilo e Mascareño, 2005, p. 33). Sin embargo, para autores como Rovira:

Un nexo evidente - aunque curiosamente aún no investigado - entre el concepto de dependencia y la globalización es el acento que ambos ponen en el problema del nacionalismo metodológico, esto es: la crítica a la suposición heurística de que sociedad y estado nación son una misma unidad 
de análisis. Tanto las teorías de la dependencia como las de la globalización tienden a objetar la idea de que una sociedad se organiza tan sólo en función de sus fuerzas internas y, a su vez, tienden a rebatir la tesis de que el estado nación sea una forma natural, pacífica, simple y única de organización social. (Rovira, 2005, p. 66)

Por lo demás, según el autor citado anteriormente, el proceso de globalización en parte re-actualizaría la discusión sobre soberanías nacionales y nuevas formas de subordinación internacional. La historia y el presente latinoamericano se caracterizarían por una contradicción entre la paulatina consecución de progresos sociales y la reproducción de mecanismos de subordinación en el orden mundial. Al mismo tiempo, Rovira toma el cuidado de afirmar que la similitud entre ambos debates es parcial, pues los desafíos de la nueva interconexión mundial serían de otro orden.

Un autor como Martín Hopenhayn (1994) no dudará en reivindicar, incluso, el antecedente inmediato de las Teorías de la Dependencia: las teorías cepalianas. Para él no se puede descartar a priori el arsenal interpretativo del enfoque estructural del capitalismo periférico. No sólo por el potencial de éste para generar capacidad crítica y constructiva en relación a los estilos de modernización en la región en el pasado, sino, sobre todo, porque muchas de las advertencias y sospechas continúan siendo actuales, tales como: la insuficiencia dinámica de acumulación del capitalismo latinoamericano, el desequilibrio en la relación crecimientoequidad y la aún persistente heterogeneidad estructural del continente.

El proceso de reprimarización de las economías latinoamericanas durante el auge neoliberal desindustrializante puede ser usado como una prueba de la permanencia de lógicas de subordinación en el establecimiento de la división internacional del trabajo. Así, por lo menos, lo demuestra Alice Amsden (2001) quien, en su libro The Rise of the rest, nos proporciona un marco analítico original, aunque, no obstante, coherente, 
a grandes rasgos, con las ideas sobre dependencia. Esta autora establece la distinción entre the rest, por un lado, o sea, aquellos países que adquirieron una experiencia manufacturera suficiente en la producción de commodities tales como algodón, seda, alimentos y bienes de consumo leves y que se desplazaron a sectores de tecnología media y alta, y the reminder, por otro, que considera a aquellos países que no consiguieron familiarizarse con formas manufactureras en el periodo pre-guerra y que están lejos de la diversificación productiva de the rest.

Para Amsden, el desarrollo económico es un proceso de desplazamiento a partir de un grupo de ventajas basadas en productos primarios, explotados por trabajo no calificado, para un set de ventajas basadas en el conocimiento, explotado por trabajo calificado. En ese proceso, los países con industrialización tardía, the rest, dieron un gran paso, pero que suponía la total dependencia de otros países en la comercialización de tecnologías para establecer industrias modernas. Al interior de the rest, se estableció una nueva distinción fundamental, pero ahora entre "compradores" (Buying) y "productores" (Making) de tecnología. Así, no obstante todos los países del resto inicialmente compraron más que produjeron tecnología, durante los años 90 se produjo una gran brecha por los gastos en ventajas basadas en conocimiento entre países productores versus países compradores de tecnología. A pesar de que entre 1950 y 1980 todos los países del resto (con excepción de Argentina) compartían un nivel de industrialización, productividad y fortaleza institucional similares, las exigencias para éstos fueron diferentes a partir de los años 80, donde los requerimientos tecnológicos van a ser decisivos para la inserción en la economía mundial.

La opción hecha por los países latinoamericanos por gerenciar economías desindustrializadas y reprimarizadas, los situará en el eje de los países "compradores" de tecnología. Esta elección por una posición subordinada no hubiera sido posible sin la expansión en América Latina 
del consenso de Washington, que llevo a un auge del neoliberalismo en el continente. De esta manera, la trayectoria económica latinoamericana podría ser explicada por el ciclo, según la fórmula de Paul Singer (1998), que establece el paso de una dependencia consentida (en los comienzos de las repúblicas), a una (mal) tolerada (período desarrollista), para llegar finalmente a una dependencia deseada (período neoliberal). Tomando como referencia esta formulación, el propio Enzo Faletto señalará, en un sugerente artículo titulado De la Teoría de la Dependencia al Proyecto Neoliberal: el caso chileno, que la temática de la dependencia es un tipo de reflexión que, con adecuaciones mediante, puede perfectamente alumbrar aspectos concretos de la particularidad que adquiere la globalización en los países latinoamericanos, toda vez que este proceso se vincula con una nueva forma de dependencia signada por un capitalismo globalizado y con fuerte predominio de las multinacionales (Faletto, 2008, p. 65).

Otros debates han substituido a la dependencia en la discusión teórica regional, a saber, globalización, ciudadanía, etc. Mas, no parece claro que las condiciones de dependencia hayan desaparecido totalmente, sea en términos económicos, políticos e incluso de producción de conocimiento. Pareciera que la ausencia del debate actual de esta teoría responde más al declive de los proyectos políticos que se identificaron con su conceptualización para comprender y transformar la realidad, que a las debilidades que pueda observar su propia arquitectura teórica. Tal vez la particular trayectoria de uno de sus más celebres exponentes, Fernando Henrique Cardoso, sea un buen reflejo de esto (Velasco e Cruz, 1999). Para muchos, la práctica política de Cardoso representa una especie de abdicación de la teoría o, como le gusta decir a sus enemigos políticos, cuando Cardoso asumió la presidencia dejó de analizar la dependencia para practicarla. Como sea, la respuesta ante las interrogantes que generaba la relación entre el pensamiento previo y el actuar de este intelectual, 
una vez que asumió la primera magistratura de Brasil, pareció sentenciar el destino de esta teoría: esqueçam tudo que eu escrevi.

\section{Roger Vekemans y la Marginalidad en América Latina}

Diversas teorías utilizaron la categoría de "marginalidad" como variable explicativa fundamental para dar cuenta de la especificidad de América Latina. Los diversos énfasis (productivos, de organización técnica de la producción, socioculturales, políticos, demográficos) mostraron que la discusión de la marginalidad no puede ser aislada de los supuestos relativos a los contrastantes modelos de desarrollo que buscaban ser alternativa de comprensión y de superación del subdesarrollo (Germani, 1973a).

Pese a esta heterogeneidad teórica, las diferentes perspectivas sobre la marginalidad poseían en común la lectura de disfuncionalidad de la misma e, independiente de la matriz ideológica, asociaban las camadas marginales con un potencial disruptivo, fuera positivamente por su capacidad revolucionaria o negativamente por la carga que representaban para el resto de la sociedad. Bajo esta noción está supuesta la necesidad de cambios en la sociedad, fueran revolucionarios o reformistas (Machado da Silva, 1971).

En este marco emerge la figura de Roger Vekemans, sociólogo belga y sacerdote jesuita, quien fuera uno de los principales teóricos de la marginalidad en el continente. Estando políticamente ligado a la Democracia Cristiana, realizó su reflexión, principalmente, desde el Centro para el Desarrollo Social para América Latina (DESAL) con sede en Santiago de Chile. Para autores como Lezama (2002) la importancia de la Teoría de la Marginalidad para la consolidación de diversas corrientes de pensamiento que buscaron pensar la especificidad del desarrollo urbano en América Latina de una manera sistemática fue fundamental. 
Desde la mirada de Vekemans, la marginalidad se diferenciaba de la pobreza en su radicalidad, pues suponía una distinción fundamental entre un sector participante y un sector marginado, en donde la imagen de un centro/periferia está presente, no sólo en el ámbito de las relaciones internacionales, sino al interior de las propias sociedades. Es por ello que, bajo esta lógica, ni la categoría de "subdesarrollo", ni la de "dependencia" daban cuenta de la especificidad de América Latina. Para este autor, el problema era que no existía una cohesión suficiente para que las sociedades constituyesen un conjunto. América Latina sería una unidad social que como totalidad estaría en "devenir", pero con una ruptura no resuelta. Para comprender esto sería indispensable cruzar una dimensión vertical del análisis de estratificación con la horizontalidad de un "adentro" y "afuera" internalizado en la sociedad.

La marginalidad sería así la falta de participación y de pertenecimiento a la sociedad, siendo lo propio de América Latina una dicotomía entre una sociedad participante versus una sociedad de masas marginales. Por ello, la marginalidad sería relacional y negativa (carencia), pues el marginal está fuera de las decisiones que modelan la sociedad y de las estructuras con las que ella se crea a sí misma. En las palabras de este autor, el mundo marginal (...) es un mundo internamente desintegrado, atomizado, un mundo en el que si se encuentran "coagulaciones" ellas serán del tipo ghetto, replegadas sobre sí mismas, a la defensiva, y no dispuestas a enfrentarse con la sociedad establecida (Vekemans e Silva, 1976, p. 81).

La marginalidad para Vekemans, a diferencia de autores como Quijano (1970), no es un subproducto de la dependencia; para aquél, la dependencia agrava la marginalidad, pero no la crea. La marginalidad contribuye a generar la dependencia, porque nuestros países estarían constituidos por un "afuera" transatlántico, internalizado en un adentro por parte de las burguesías "herodianas" locales, las que seguirían vivien- 
do en una matriz externa, manteniendo una orientación hacia "afuera", fortaleciendo la escisión interna.

Así, el continente sólo tomó consciencia de la marginalidad preexistente cuando se produjo la avalancha sobre las "urbes", o sea, en el momento en que físicamente fue posible mostrar con el dedo los cordones de miseria (tugurios, barriadas, callampas, favelas). Esta noción explica el fuerte tono de advertencia de esta teoría frente a la amenaza de los marginales: La masa marginal se encuentra, metafóricamente, frente a la vitrina pero sin poder de compra (...) Han pasado cuatro siglos y frente a este nuevo hecho es necesario actuar para que la vitrina no estalle violentamente (Vekemans e Silva, 1969, p. 61).

Las Teorías de la Marginalidad recibieron un fuerte cuestionamiento en la aplicación de sus hipótesis en el mundo empírico por parte de diversos autores. Una serie de investigaciones mostraron que las premisas de la marginalidad no tenían sustento, pues, la pobreza urbana se mostraba más heterogénea de lo que anunciaba la imagen de "masa marginal", además de mostrar que los "marginados" tenían otras fuentes de integración a la ciudad: trabajo, sindicatos, religión, educación de los hijos, etc. (CIDU, 1972; Pastrana e Threalfall, 1974; Pearlman, 1977; Oliven, 1980; Sabatini e Worlmald, 2004).

No obstante buena parte de los más reconocidos investigadores de la cuestión urbana en América Latina se formó debatiendo, criticando y deconstruyendo los principales supuestos e hipótesis de la Teoría de la Marginalidad, en el último tiempo es posible observar una fuerte tendencia de reaparición de tópicos propios de la marginalidad. Incluso así, no deja de sorprender que uno de los principales críticos a ella en los años 70, Manuel Castells (1972), no hace mucho haya afirmado que si la marginalidad urbana era un mito cuando se formuló su teoría en los setenta (puesto que la mayor parte de los llamados marginales urbanos estaban 
integrados en la economía formal) en estos momentos sí es una realidad masiva (Castells, 2005, p. 35).

A comienzos de la década de los 80, Machado da Silva (1983) ya llamaba la atención para el hecho de que, a pesar de que la mayoría de los cientistas sociales consideraban que la Teoría de la Marginalidad estaba muerta y enterrada, en la práctica continuaba viva, pues el modelo que la corporificaba aún era usado en las políticas sociales y en los discursos de actores políticos y medios de comunicación; al mismo tiempo, continuaba insepulta, ya que el mundo académico aún no había completado la tarea de reordenamiento sistemático del problema.

Sumado a lo anterior, será la emergencia y fortalecimiento de una serie de estudios sobre la "exclusión social" lo que en buena parte resucitará a la Marginalidad como categoría útil de comprensión. En esa línea, será José Nun (2001) el autor que más enfatizará el hecho de que muchas de las problemáticas levantadas por las teorías de la exclusión en Europa retoman, y en algunos casos replican, tópicos abordados por la marginalidad en los años 60. Patrick Cingonali (2009) en un artículo relativamente reciente intentó mapear el intercambio transatlántico de las ideas relacionadas con la marginalidad para dar cuenta del fenómeno de aumento de poblaciones disfuncionales frente al régimen de producción dominante, particularmente en los suburbios de los países desarrollados.

De la misma manera, Saraví (2006) afirma que las teorías de la marginalidad comparten con las nuevas perspectivas sobre la exclusión, la preocupación por la falta de integración de sectores específicos de la población, entendiendo por exclusión, el resultado final de un proceso de acumulación de desventajas que corroe la relación individuo-sociedad y con ella las condiciones de pertenecimiento y de solidaridad mismas. Aunque esto acontecería en un marco que presenta rupturas significativas frente a la realidad del siglo XX: disminución de la capacidad de asocia- 
ción y gestión colectiva de los sectores populares, endurecimiento de la estructura social, segregación espacial de la pobreza, debilitamiento del Estado y de sus políticas de intervención social, entre otras.

Resulta paradojal que la teoría que aparentemente presentaba una menor densidad teórica de las expuestas aquí, la que recibió más críticas y la que tuvo que lidiar con la negación de sus hipótesis centrales, sea la que se muestra más "saludable" actualmente. ¿Qué puede explicar esta vigencia inesperada? Aunque la respuesta a esta interrogante, así como su valoración (positiva o negativa), superan la finalidad de este trabajo, un buen comienzo para ensayar una réplica es la reflexión que el propio Germani (1973a) elaboró al aseverar que la marginalidad era la falta de participación en esferas que se deberían considerar como mínimas para la integración de los individuos, o sea, la idea de marginalidad se erguía a partir de la comparación de una situación de hecho y una situación ideal (deber ser). De esta manera, lo que podría ser un obstáculo normativo, fue la clave universalista que le otorgó un grado de actualidad más allá de su propio tiempo.

\section{Consideraciones Finales}

Una de las expectativas de este trabajo fue consolidar la idea de que el desafío de crear una teoría sociológica sensible a los contextos latinoamericanos, pero que no termine en un latinoamericanismo particularista, continua vigente (Domingues, 2007). De la misma manera, y pese a que existen nuevas tendencias que afirman la ruptura con la tradición sociológica anterior, como es el caso de las perspectivas posmodernas, se hace necesario remarcar que, siguiendo a Hopenhayn (1994), la crítica a la direccionalidad normativa de la historia de nuestro continente y del pensa- 
miento social no se debe confundir con el rechazo de toda direccionalidad, en tanto la necesidad colectiva de imágenes de futuro continua presente.

Es por ello que la relectura de la teoría latinoamericana del siglo XX, y más aún de clásicos como Germani, Cardoso y Faletto, es una tarea imprescindible. Es más, algunos de los elementos centrales de las teorías brevemente esbozadas aquí podrían ser reactualizados por nuevas discusiones. Así, por ejemplo, la Teoría de Sistema podría revivir tópicos de la contribución germaniana por la noción de transición permanente implícita en el proceso de especialización funcional de la primera. Por otro lado, el proceso de globalización en parte actualiza la discusión sobre soberanías nacionales y nuevas formas de subordinación internacional, además de tener en común con la teoría de la dependencia el rechazo por explicaciones basadas en el nacionalismo metodológico. Finalmente, las teorías sobre la Exclusión Social nacen debatiendo en continuidad y ruptura con las Teorías de la Marginalidad.

La sucinta exposición de estas tres teorías, pese a sus limitaciones, sirvió para mostrar que, en particular en el caso de Germani y de Cardoso y Faletto, señalarlas como meras representantes de una "sociología de la incompletitud" que comprende América Latina como una copia infeliz de los países industrializados (Chernilo e Mascareño, 2005) es, al menos, una reducción injusta. Por más que, por ejemplo en el caso de Germani, la pregunta por el tránsito a la modernización esté superada - por lo menos en los términos en que se elaboró en su época -, la reflexión de este autor se ha mostrado fecunda y bien más compleja, obviamente, que las simplificaciones que han circulado sobre su teoría. La relectura de la obra de los autores estudiados aquí no sólo es indispensable para entender la evolución de la teoría social elaborada en nuestro continente, como puede ofrecer luces que enriquezcan aún más nuestra comprensión de lo que hoy es América Latina. 
Por otro lado, el permanente desafío representado por la "falta de autonomía del campo sociológico" por la subordinación de la dimensión descriptiva a la normativa, aludida por Chernilo y Mascareño, debe ser cualificada. Como vimos, la marcada adscripción política de autores ligadas a la Teoría de la Marginalidad - en el caso de este texto, Vekemans - no impidió la fértil reproducción de sus supuestos, aunque de manera indirecta, en las actuales discusiones sobre exclusión social. La opción política de la versión de la Teoría de la Marginalidad expuesta aquí no le quitó actualidad al debate que proponía. Y es que la autonomía intelectual no es sinónimo de falta posicionamiento en el campo teórico y político. Y, aunque se debe reconocer que un gran número de autores limitaron su alcance teórico al confundir compromiso intelectual con reproducción mecánica de hipótesis predefinidas en ciertas teorías, en nuestros clásicos latinoamericanos existe un buen ejemplo de cómo orientar la creación en el campo. Tómense como referencia, una vez más, las palabras de Germani:

Una clara conciencia teórica en cuanto a las implicaciones ideológicas del propio pensamiento y una actitud vigilante orientada exclusivamente en la búsqueda de la verdad constituyen dos condiciones esenciales de todo quehacer científico. La imparcialidad absoluta es quizá tan sólo una meta ideal hasta cierto punto inalcanzable, pero la honestidad moral y la claridad intelectual (...) son calidades indispensables para el investigador. (Germani, 1995, p. 17)

La revisión teórica realizada en este trabajo deja una conclusión clara. El desconocimiento, la incomprensión o la simplificación de nuestros clásicos latinoamericanos, antes que cualquier otro, pueden llegar a ser el primer gran "obstáculo epistemológico" para avanzar en la construcción de una teoría sociológica que dé cuenta de la complejidad de nuestro continente. 


\section{Referencias}

ACEVEDO RODRÍGUEZ, Carlos. Germani y el Estructural Funcionalismo, Evolucionismo y Fe en la Razón: aspectos de la involución irracional. Universum (Talca), v. 24, n. 1, p. 12-20, I Semestre, 2009.

AMSDEN, Alice. The Rise of "The Rest": challenges to the west from late-industrializing economies. Oxford: Oxford University Press, 2001.

BLANCO, Alejandro. Política, Modernización y Desarrollo: una revisión de la recepción de Talcott Parsons en la obra de Gino Germani. Estudios Sociológicos, v. XXI, n. 003, p. 667-699, 2003.

CARDOSO, Fernando Henrique; FALETTO, Enzo. Dependencia y Desarrollo en América Latina: ensayo de interpretación sociológica. Buenos Aires: Siglo XXI, 2003.

CASTELLS, Manuel. Chile: movimiento de pobladores y lucha de clases. Santiago: Mimeo, 1972.

CASTELLS, Manuel. Globalización, desarrollo y democracia: Chile en el contexto mundial. Santiago: Fondo de Cultura Económica, 2005.

CIDU. Reivindicación Urbana y Lucha Política: los campamentos de pobladores en Santiago de Chile. EURE. v. 2, n. 6, p. 55-82, noviembre, 1972.

CINGOLANI, Patrick. Marginalidad(es). Esbozo de diálogo Europa-América Latina acerca de una categoría sociológica. Revista Latinoamerciana de Estudios del Trabajo. v. n. 22, p. 157-166, 2 semestre, 2009.

CHERNILO, Daniel; MASCAREÑO, Aldo. Universalismo, Particularismo y Sociedad Mundial: obstáculos y perspectivas de la sociología de América Latina. Persona y Sociedad. v. XIX, n. 3, p. 17-45, 2005.

DOMINGUES, José Maurício. Aproximações à América Latina: desafios contemporâneos. Rio de Janeiro: Civilização Brasileira, 2007.

DOMINGUES, José Maurício; MANEIRO, María. Revisitando Germani: a interpretação da modernidade e a teoria da ação. DADOS. v. 47, n. 4, p. 643-668, 2004.

FAlETTO, Enzo. Dimensiones Sociales, Políticas y Culturales del Desarrollo. Santiago: FLACSO CHILE / Catalonia, 2008.

GERMANI, Gino. Sociología de la Modernización: estudios teóricos, metodológicos y aplicados a América Latina. Buenos Aires: Paidós, 1971.

GERMANI, Gino. El Concepto de Marginalidad: significado, raíces históricas y cuestiones teóricas, con particular referencia a la marginalidad urbana. Buenos Aires: Nueva Visión, 1973a. 
GERMANI, Gino. Política e Sociedade numa época de transição: da sociedade tradicional à sociedade de massas. São Paulo: Mestre Jou, 1973b.

GERMANI, Gino. Prólogo a la Imaginación Sociológica. In: MILLS, C. Wirght. La Imaginación Sociológica. México: FCE, 1995.

HOPENHAYN, Martín. Ni Apocalípticos ni Integrados: aventuras de la modernidad en América Latina. México: FCE, 1994.

IZAGUIRRE, Inés. Acerca de un maestro. Gino Germani, fundador de la sociología en Argentina. Sociologias, n. 14, p. 492-503, jun/dez 2005.

LARRAÍN, Jorge. Theories of Development: capitalism, colonialism and dependency. Cambridge: Polity Press, 1989.

LEZAMA, José Luis. Teoría Social, Espacio y Ciudad. México: Colegio de México, 2002.

MACHADO DA SILVA, Luiz Antonio. Mercados de Trabalho Manual e Marginalidade, 1971. Dissertação (Mestrado em Antropologia) - Programa de PósGraduação em Antropologia Social, UFRJ, Rio de Janeiro, 1971.

MACHADO DA SILVA, Luiz Antonio. Vida e Morte da Teoria da Marginalidade. In: KONDER, L.; CERQUEIRA FILHO, G.; FIGUEIREDO, E. L. (Eds.). Por Que Marx? Rio de Janeiro: Graal, 1983. p.217-232.

MASCAREÑO, Aldo. Sociología del Derecho (Chileno y Latinoamericano). Persona y Sociedad, v. 18, n. 2, p. 63-94, 2004.

MOORE, Barrington. As Origens Sociais da Ditadura e da Democracia: senhores e camponeses na construção do mundo moderno. Lisboa: Cosmos, 1975.

MORANDÉ, Pedro. Cultura y modernización en América Latina: ensayo sociológico acerca de la crisis del desarrollismo y de su superación. Madrid: Encuentro Ediciones, 1987.

NUN, José. Marginalidad y Exclusión Social. Buenos Aires: Fondo de Cultura Económica, 2001.

OLIVEN, Ruben George. Marginalidad Urbana en América Latina. EURE, v. 7, n. 19, p. 49-62, octubre 1980.

PASTRANA, Ernesto; THREALFALL, Mónica. Pan, techo y poder: el movimiento de pobladores en Chile (1970-1973). Buenos Aires: Ediciones Siap, 1974.

PEARLMAN, Janice. O Mito da Marginalidade: favelas e política no Rio de Janeiro. Rio de Janeiro: Paz e Terra, 1977.

PREBISCH, Raúl. El Desarrollo Económico de la América Latina y sus principales problemas. Naciones Unidas Consejo Económico y Social, 1947. 
QUIJANO, Aníbal. Redefinición de la Dependencia y Proceso de Marginalización en América Latina. Santiago: OIT-ILPES, 1970. Mimeo.

ROVIRA, Cristóbal. Globalización en América Latina: sobre los aportes y perjuicios sociales de la dependencia. Persona y Sociedad, v. XIX, n. 3, p. 63-84, 2005.

SABATINI, Francisco; WORLMALD, Guillermo. La guerra de la basura de Santiago: desde el derecho a la vivienda al derecho a la ciudad. EURE, v. XXX, n. 91, p. 67-86, diciembre 2004.

SARAVÍ, Gonzalo. Nuevas realidades y nuevos enfoques: exclusión social en América Latina. In: SARAVÍ, Gonzalo (Ed.). De la pobreza a la exclusión: continuidades y rupturas de la cuestión social en América Latina. Buenos Aires-México: CIESAS-Prometeo, 2006.

SINGER, Paul. De dependência em dependência: consentida, tolerada e desejada. Estudos Avançados, v. 12, n. 33, p. 119-130, Mai/Ago, 1998.

VÁRELA PETITO, Gonzalo. Gino Germani en su circunstancia. Perfiles latinoamericanos, v. 16, n. 32, p. 235-243, jul./dic, 2008.

VEKEMANS, Roger; SILVA, Ismael. El Concepto de Marginalidad. In: DESAL. Marginalidad en América Latina: un ensayo de diagnóstico. Santiago: Herder, 1969. p.15-63

VEKEMANS, Roger; SILVA, Ismael. Marginalidad, Promoción Popular y NeoMarxismo. Bogotá: Cedial, 1976

VELASCO E CRUZ, Sebastião Carlos. Idéias do poder: dependência e globalização em F. H. Cardoso. Estudos Avançados, v. 13, n. 37, p. 225-247, Set./Dez. 1999.

Recebido em: 28/04/2011

Aceite final: 06/09/2011 